\title{
Small world effect in an epidemiological model
}

\author{
Guillermo Abramson ${ }^{1,2} *$ and Marcelo Kuperman ${ }^{1} \dagger$ \\ ${ }^{1}$ Centro Atómico Bariloche and Instituto Balseiro, 8400 S. C. de Bariloche, Argentina \\ ${ }^{2}$ Consejo Nacional de Investigaciones Científicas y Técnicas, Argentina
}

A model for the spread of an infection is analyzed for different population structures. The interactions within the population are described by small world networks, ranging from ordered lattices to random graphs. For the more ordered systems, there is a fluctuating endemic state of low infection. At a finite value of the disorder of the network, we find a transition to self-sustained oscillations in the size of the infected subpopulation.

PACS numbers: 87.23.Ge, 05.65.+b, 87.19.Xx

\section{INTRODUCTION}

How does the dynamics of an infectious disease depend on the structure of a population? A great amount of work has been done on the phenomenological description of particular epidemic situations 플. A classical mathematical approach to these problems deals with well mixed populations, where the subpopulations involved (typically susceptible, infected and removed) interact in proportion to their sizes. With these zero dimensional models it has been possible to study, among other epidemic features, the existence of threshold values for the spread of an infection [5], the asymptotic solution for the density of infected people [6-8], the effect of stochastic fluctuations on the modulation of an epidemic situation [9]. A second classical approach describes spatially extended subpopulations. In this, the geographic spread of an epidemic can be analyzed as a reaction-diffusion process 10 12.

Real populations rarely fall into either of these categories, being neither well mixed nor lattices. Recently introduced by Watts and Strogatz [13], small world networks attempt to translate, into an abstract model, the complex topology of social interactions. Small worlds may play an important role in the study of the influence of the network structure upon the dynamics of many social processes, such as disease spreading, formation of public opinion, distribution of wealth, transmission of cultural traits, etc. [14]. In relation to epidemiological models, it has been shown that small world networks present a much faster epidemic propagation than

${ }^{*}$ E-mail address: abramson@cab.cnea.gov.ar

${ }^{\dagger}$ E-mail address: kuperman@cab.cnea.gov.ar reaction-diffusion models or discrete models based on regular lattices of a social network 15 .

In the original model of small worlds a single parameter $p$, running from 0 to 1 , characterizes the degree of disorder of the network, respectively ranging from a regular lattice to a completely random graph. It has been shown that geometrical properties, as well as certain statistical mechanics properties, show a transition at $p_{c}=0$ in the limit of large systems, $N \rightarrow \infty$ [16]. That is, any finite value of the disorder induces the small world behavior. In this article we show that a sharp transition in the behavior of an infection dynamics exists at a finite value of $p$.

\section{EPIDEMIC MODEL}

We analyze a simple model of the spread of an infectious disease. We want, mainly, to point to the role played by the network structure on the temporal dynamics of the epidemic. The disease has three stages: susceptible $(S)$, infected $(I)$, and refractory $(R)$. An element of the population is described by a single dynamical variable adopting one of these three values. Susceptible elements can pass to the infected state through contagion by an infected one. Infected elements pass to the refractory state after an infection time $\tau_{I}$. Refractory elements return to the susceptible state after a recovery time $\tau_{R}$. This kind of system is usually called $S I R S$, for the cycle that a single element goes over. The contagion is possible only during the $S$ phase, and only by an $I$ element. During the $R$ phase, the elements are immune and do not infect.

The interactions between the elements of the population are described by a small world network. The links represent the contact between subjects, and infection can only proceed through them. As in Watts and Strogatz model, the small worlds we study are random networks built upon a topological ring with $N$ vertices and coordination number $2 K$. Each link connecting a vertex to a neighbor in the clockwise sense is then rewired at random, with probability $p$, to any vertex of the system. With probability $(1-p)$ the original link is preserved. Self-connections and multiple connections are prohibited. With this procedure, we have a regular lattice at $p=0$, and progressively random graphs for $p>0$. The long range links that appear at any $p>0$ trigger the small world phenomenon. At $p=1$ all the links have been rewired, and the result is similar to (though not exactly) a completely random network. This algorithm should be used with caution, since it can produce disconnected 
graphs. We have used only connected ones for our analysis.

Time proceeds by discrete steps. Each element is characterized by a time counter $\tau_{i}(t)=0,1, \ldots, \tau_{I}+\tau_{R} \equiv \tau_{0}$, describing its phase in the cycle of the disease. The epidemiological state $\pi_{i}$ of the element $(S, I$ or $R$ ) depends on this phase in the following way:

$$
\begin{array}{ll}
\pi_{i}(t)=S & \text { if } \tau_{i}(t)=0 \\
\pi_{i}(t)=I & \text { if } \tau_{i}(t) \in\left(1, \tau_{I}\right) \\
\pi_{i}(t)=R & \text { if } \tau_{i}(t) \in\left(\tau_{I}+1, \tau_{0}\right)
\end{array}
$$

The state of an element in the next step depends on its current phase in the cycle, and the state of its neighbors in the network. The rules of evolution are the following:

$$
\begin{array}{ll}
\tau_{i}(t+1)=0 & \text { if } \tau_{i}(t)=0 \text { and no infection occurs } \\
\tau_{i}(t+1)=1 & \text { if } \tau_{i}(t)=0 \text { and } i \text { becomes infected } \\
\tau_{i}(t+1)=\tau_{i}(t)+1 & \text { if } 1 \leq \tau_{i}(t)<\tau_{0} \\
\tau_{i}(t+1)=0 & \text { if } \tau_{i}(t)=\tau_{R}
\end{array}
$$

That is, a susceptible element stays as such, at $\tau=0$, until it becomes infected. Once infected, it goes (deterministically) over a cycle that lasts $\tau_{0}$ time steps. During the first $\tau_{I}$ time steps, it is infected and can potentially transmit the disease to a susceptible neighbor. During the last $\tau_{R}$ time steps of the cycle, it remains in state $R$, immune but not contagious. After the cycle is complete, it returns to the susceptible state.

The contagion of a susceptible element by an infected one, and the subsequent excitation of the disease cycle in the new infected, occur stochastically at a local level. Say that the element $i$ is susceptible, and that it has $k_{i}$ neighbors, of which $k_{\text {inf }}$ are infected. Then, $i$ will become infected with probability $k_{\text {inf }} / k_{i}$. Observe that $i$ will become infected with probability 1 if all its neighbors are infected. There are other reasonable choices for this mechanism. For example, if the susceptible had a probability $q$ of contagion with each infected neighbor, we would have a probability of infection $\left[1-(1-q)^{k_{\mathrm{inf}}}\right]$. We have tested that both these criteria give qualitatively the same results for values of $q \lesssim 0.2$.

\section{NUMERICAL RESULTS}

We have performed extensive numerical simulations of the described model. Networks with $N=10^{3}$ to $10^{6}$ vertices have been explored, with $K=3$ to 10 . A typical realization starts with the generation of the random network and the initialization of the state of the elements. An initial fraction of 0.1 infected, and the rest susceptible, was used in all the results shown here. Other initial conditions have been explored as well, and no changes have been observed in the behavior. After a transient a stationary state is achieved, and the computations are followed for several thousand time steps to perform statistical averages.

We show in Fig. 1 part of three time series displaying the fraction of infected elements in the system, $n_{\text {inf }}(t)$. The three curves correspond to systems with different values of the disorder parameter: $p=0.01$ (top), 0.2 (middle) and 0.9 (bottom). The three systems have $N=10^{4}$ and $K=3$, and infection cycles with $\tau_{I}=4$ and $\tau_{R}=9$. The initial state is random with $n_{\text {inf }}(0)=0.1$. The 400 time steps shown are representative of the stationary state. We can see clearly a transition from an endemic situation to an oscillatory one. At $p=0.01$ (top), where the network is nearly a regular lattice, the stationary state is a fixed point, with fluctuations. The situation corresponds to that of an endemic infection, with a low and persistent fraction of infected individuals. At high values of $p$-like the case with $p=0.9$ shown in the figure (bottom) - large amplitude, self-sustained oscillations develop. The situation is almost periodic, with a very well defined period and small fluctuations in amplitude. The period is slightly longer than $\tau_{0}$, since it includes the average time that a susceptible individual remains at state $S$, before being infected. Epidemiologically, the situation resembles the periodic epidemic patterns typical of large populations [3]. A mean field model of the system, expected to resemble the behavior at $p=1$, can be easily shown to exhibit these oscillations. The transition between both behaviors is apparent-in this relatively small system - at the intermediate value of disorder $p=0.2$, shown in the middle curve. Here a low amplitude periodic pattern can be seen, appearing and disappearing again in a background of strong fluctuations. Moreover, the mean value of infection is seen to grow with $p$.

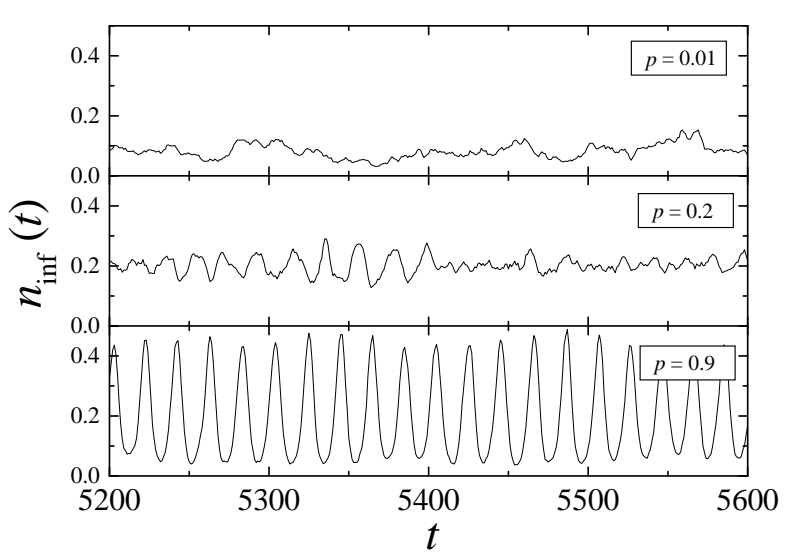

FIG. 1. Fraction of infected elements as a function of time. Three time series are shown, corresponding to different values of the disorder parameter $p$, as shown in the legends. Other parameters are: $N=10^{4}, K=3, \tau_{I}=4, \tau_{R}=9$, $N_{\text {inf }}(0)=0.1$.

The formation of persistent oscillations corresponds to a spontaneous synchronization of a significant fraction of 
the elements in the system. Their phases $\tau_{i}(t)$ in the epidemic cycle become synchronized, and they go over the disease process together, becoming ill at the same time, and recovering at the same time. We can characterize this behavior with a synchronization parameter [17], defined as:

$$
\sigma(t)=\left|\frac{1}{N} \sum_{j=1}^{N} e^{i \phi_{j}(t)}\right|,
$$

where $\phi_{j}=2 \pi\left(\tau_{j}-1\right) / \tau_{0}$ is a geometrical phase corresponding to $\tau_{j}$. We have chosen to let the states $\tau=0$ out of the sum in (3), and take into account only the deterministic part of the cycles.

When the system is not synchronized, the phases are widely spread in the cycle and the complex numbers $e^{i \phi}$ are correspondingly spread in the unit circle. In this situation $\sigma$ is small. On the other hand, when a significant part of the elements are synchronized in the cycle, $\sigma$ is large. The synchronization would be strictly $\sigma=1$ if all the elements were at the same state at the same time. However, such a state would end up in $N_{\text {inf }}=0$ after $\tau_{0}$ time steps, and the epidemic would end since the system is closed and no spontaneous infection of susceptibles is being taken into account in the model.

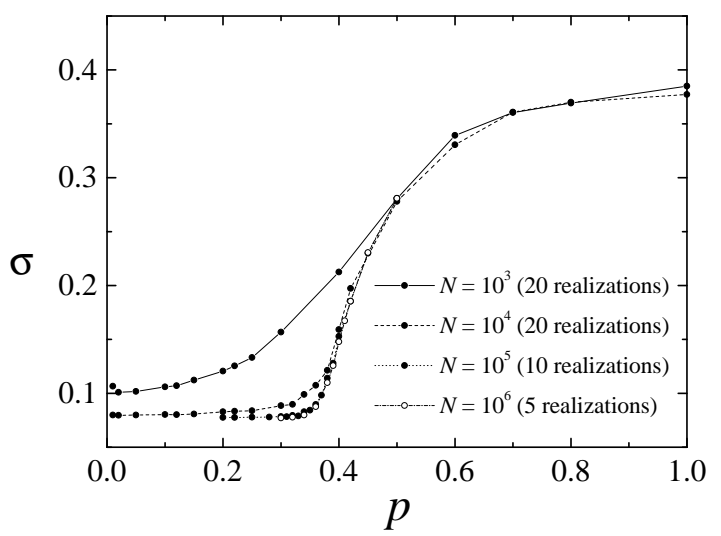

FIG. 2. Synchronization of the system as a function of the disorder parameter $p$. Three curves are shown, corresponding to different system sizes $N=10^{3}, N=10^{4}, N=10^{5}$ and $10^{6}$, as shown in the legend. Each point corresponds to a time average of 2000 time steps, and a subsequent average over a number of realizations of the networks and the initial condition, as shown in the legend. Other parameters are: $K=3, \tau_{I}=4, \tau_{R}=9, N_{\mathrm{inf}}(0)=0.1$.

In Fig. 2 we show the synchronization parameter $\sigma$, obtained as a time average of $\sigma(t)$ over 2000 time steps and a subsequent average over realizations of the system. Several curves are shown, corresponding to system sizes $N=10^{3}, 10^{4}, 10^{5}$ and $10^{6}$. A transition in the synchronization can be seen as $p$ runs from 0 to 1 . The transition becomes sharper for large systems, at a value of the disorder parameter $p_{c} \approx 0.4$. It is worthwhile to remark that the transition to synchronization occurs as a function of the structure of the network, contrasting the phenomenon of synchronization as a function of the strength of the interaction, as in other systems of coupled oscillators 18]. This behavior is observed for a wide range of values of $\tau_{I}$ and $\tau_{R}$. The amplest oscillations take place around $\tau_{I} / \tau_{R}=1$. They disappear when $\tau_{I}$ is significantly greater or smaller than $\tau_{R}$.

All the systems shown in figures 1 and 2 have $K=3$. We have explored higher values of $K$ as well. The picture is qualitatively the same, with a sharp transition from a quasi-fixed point to a quasi-limit cycle at a finite value of $p$. The critical value $p_{c}$ shifts toward lower values for growing $K$. This is reasonable, since higher values of $K$ approaches the system to a globally coupled one (at $K=N$ all the elements interact with every other one, even at $p=0$ ). So, the mean field behavior (the oscillations) can be expected to occur at lower values of $p$. In figure 3 this effect can be seen for a system with $N=10^{4}$ and growing values of $K=3,5$ and 10.

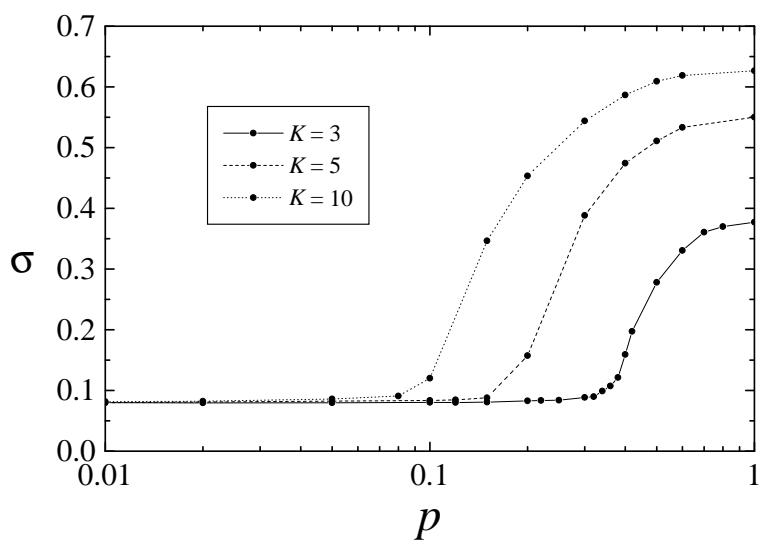

FIG. 3. Synchronization of the system as a function of the disorder parameter $p$. Three systems with different values of the coordination number $K$ are shown. All of them have $N=10^{4}, \tau_{I}=4, \tau_{R}=9, N_{\text {inf }}(0)=0.1$.

\section{DISCUSSION}

Why does this transition to synchronization take place? Unfortunately we do not have analytical arguments to describe this yet. As mentioned before, a mean field model can be shown to predict the oscillations, but this can only be expected to describe the network at $p=1$, and it does not shed light on the nature of the transition at lower values of disorder. We can only advance some conjectures, based in our observation of the dynamical behavior of the system in real time on the computer screen.

An explanation involving $L(p)$, the typical distance between pairs of elements (defined as the size of the minimum path connecting two elements) is not plausible, since $L$ is known to behave critically with $p_{c}=0$, and we 
observe the transition at $p>0$. Complementarily to $L$, small world networks can be described by the degree of clusterization $C(p)[19$. At low values of $p$, the networks are rather regular and highly clustered. As $p$ approaches 1, $C$ decreases. The crossover from high to low clusterization occurs at a higher value of $p$, compared to that observed in the decay of $L$. For systems with $K=3$, such as those mainly studied here, we have that this crossover is mostly concentrated between $p=0.1$ and $p=0.5$, precisely where the onset of oscillations occurs. Moreover, the change in the average clusterization $C(p)$ is accompanied by a corresponding one in the distribution of the clusterization at the element level, $c_{i}(p)$. Highly ordered networks, at low $p$, show not only a large value of the average clusterization $C(p)$ but also a small dispersion around it. On the other extreme, highly disordered networks, with $p>0.5$, exhibit a low average clusterization and also a low dispersion around it. There is an intermediate range of $p$, between 0.1 and 0.5 , where the average clusterization shifts from high to low, but the distributions are wide. This indicates that, in this range, the system is a mixture of highly ordered, highly clustered regions and random, lowly clustered ones. If we consider that the clustering structure determines a partition of the whole system into smaller, interacting, subsystems, the global behavior of the system could be interpreted as a superposition of the subsystems' dynamics. When $p$ is small the existence of large clusters (essentially onedimensional in our model) inhibits the oscillatory behavior because, once the infection breaks into such a region, it remains restricted to it a long time until all the individuals have completed the cycle, orderly and deterministically. As $p$ increases, the number of big, ordered regions decreases. Elements within small regions with some long range links go through the infection cycle and become infected again before long. Some degree of synchronization can be seen here (Fig. 1, center, corresponds to $p=0.2$ ). When a critical value of $p$ is reached, and the system is essentially composed of enough small regions of low clusterization and a similar local dynamics, the synchronized, periodic global behavior establishes spontaneously.

In summary, we have observed a transition at a finite value of the disorder in a small world model. The dynamical behavior of an SIRS epidemiological model changes from an irregular, low-amplitude evolution at small $p$ to a spontaneous state of wide amplitude oscillations at large $p$. This may be related to observed patterns in real epidemics [3], where an effect of the social structure is observed in the dynamics of the disease.

The authors thank D. H. Zanette for fruitful discussions.

[1] C. McEvedy, Sci. Am. (February) p. 74, (1988).
[2] M. M. Kaplan and R. G. Webster, Sci. Am., (December) p. 88, (1977).

[3] A. Cliff and P. Haggett. Sci. Am. (May) p. 110, (1984).

[4] N. Hirschhorn and W. Greenough III. Sci. Am., (August) p. 15 (1971).

[5] R. M. Anderson and R. M. May, Science 215, 1053 (1982); R. M. Anderson and R. M. May, Nature 318, 323 (1982).

[6] J. D. Murray. Mathematical Biology (Springer-Verlag, Berlin, 1993).

[7] N.T. Bailey. The mathematical theory of infectious diseases (Griffin, London, 1975).

[8] F. C. Hoppensteadt. Mathematical theories of populations: demographics, genetics and epidemics, CBMS Lectures, 20 (SIAM publications, Philadelphia, 1975).

[9] P. Landa and A. Zaikin, in Applied Nonlinear Dynamics and Stochastic Systems Near the Millennium, J. Kadkte and A. Bulsara (eds.), p. 321, (AIP 1997).

[10] J. Murray, E. Stanley and D. Brown, Proc. Roy. Soc. B229, 111 (1986).

[11] A. Källen, P. Arcury and J. Murray, J. theor. Biol. 116, 377 (1985).

[12] M. Fuentes and M. N. Kuperman, Physica A 267, 471 (1999).

[13] D. J. Watts and S. H. Strogatz, Nature 393, 440 (1998).

[14] D. J. Watts, Small Worlds (Princeton University Press, 1999).

[15] C. Moore and M. E. J. Newman, Phys. Rev. E 61, 5678 (2000).

[16] A. Barrat and M. Weigt, Eur. Phys. J. B 13, 547 (2000).

[17] Y. Kuramoto, Chemical Oscillations, Waves, and Turbulence (Springer, Berlin, 1984).

[18] J. F. Heagy, T. L. Carroll and L. M. Pecora, Phys. Rev. E 50, 1874 (1994).

[19] $C(p)$ is an average, over the system, of the local clusterization $c_{i}(p)$. This is defined as the number of neighbors, of element $i$, that are neighbors among themselves, normalized to the value that this would have if all of them were connected to one another-namely $k_{i}\left(k_{i}-1\right) / 2$. 